\title{
Erratum to: Asymmetric quantum codes: new codes from old
}

\author{
Giuliano G. La Guardia
}

Published online: 10 April 2013

(C) Springer Science+Business Media New York 2013

\section{Erratum to: Quantum Inf Process DOI 10.1007/s11128-013-0562-4}

We regret that unfortunately an error was introduced in the original version of this article. There is an error under the Section 4 Construction methods in Theorem 4.2. An inline equation was erroneously included. The correct text for Theorem 4.2 is as follows.

Theorem 4.2 Let $q$ be a prime power. Assume that there exists an AQECC with parameters $\left[\left[n, k, d_{z} / d_{x}\right]\right]_{q^{m}}$, derived from linear codes $C_{1}=\left[n, k_{1}, d_{1}\right]_{q^{m}}$ and $C_{2}=\left[n, k_{2}, d_{2}\right]_{q^{m}}$, respectively. Then, there exists an AQECC with parameters $\left[\left[m n, m k, d_{z}^{*} / d_{x}^{*}\right]\right]_{q}$, where $k=k_{1}-k_{2}, d_{z}^{*} \geq d_{1}$ and $d_{x}^{*} \geq d_{2}^{\perp}$, where $d_{2}^{\perp}$ denotes the minimum distance of the dual code $C_{2}^{\perp}$.

The online version of the original article can be found under doi:10.1007/s11128-013-0562-4.

G. G. La Guardia $(\bowtie)$

Department of Mathematics and Statistics, State University of Ponta Grossa, Ponta Grossa, PR 84030-900, Brazil

e-mail: gguardia@uepg.br 